\title{
ESTIMATES FOR ABSOLUTE VALUES OF MATRIX FUNCTIONS*
}

\author{
MICHAEL I. GIL' †
}

\begin{abstract}
Sharp estimates for the absolute values of entries of matrix valued functions of finite and infinite matrices are derived. These estimates give us bounds for various norms of matrix valued functions. Applications of the obtained estimates to differential equations are also discussed.
\end{abstract}

Key words. Matrix valued function, Estimate for entries, Estimate for norm.

AMS subject classifications. 15A54, 15A60.

1. Introduction and statement of the main result. In the book [5], I.M. Gel'fand and G.E. Shilov have established an estimate for the norm of a regular matrix valued function in connection with their investigations of partial differential equations. However that estimate is not sharp, it is not attained for any matrix. The problem of obtaining a precise estimate for the norm of a matrix function has been repeatedly discussed in the literature, cf. [1]. In the paper [6] (see also [7]) the author has derived a precise estimate for the Euclidean norm which is attained in the case of normal matrices. But that estimate requires bounds for the eigenvalues. In this paper we derive sharp estimates for the absolute values of entries of a matrix valued function. They are attained in the case of diagonal matrices. Besides, bounds for the eigenvalues are not required. These estimates give us bounds for various norms of matrix valued functions. Our results supplement the very interesting recent investigations of matrix valued functions $[3,4,9,11]$.

A few words about the contents. The paper consists of 4 sections. In this section we consider finite matrices and formulate the main result of the paper-Theorem 1.1. It is proved in Section 2. Section 3 deals with applications of Theorem 1.1 to differential equations. In Section 4 we generalize Theorem 1.1 to some classes of infinite matrices.

Let $\mathbb{C}^{n}$ be a complex Euclidean space with the scalar product (.,.) and the unit matrix $I$. Let $\sigma(A)$ be the spectrum of a linear operator (a matrix) $A$ and

$$
R_{z}(A)=(A-z I)^{-1} \quad(z \notin \sigma(A))
$$

the resolvent of $A$. For a scalar valued function $f(\lambda)$ holomorphic on the spectrum of $A$, the matrix valued function $f(A)$ is defined by

$$
f(A)=-\frac{1}{2 \pi i} \int_{\Gamma} f(\lambda) R_{\lambda}(A) d \lambda
$$

where $\Gamma$ is a closed contour surrounding $\sigma(A)$.

Furthermore, let $\left\{e_{k}\right\}_{k=1}^{n}$ be a fixed orthonormal basis in a complex Euclidean space $\mathbb{C}^{n}$, and $a_{j k}(j, k=1, \ldots, n)$ the entries of a matrix $A$ in this basis. We put

\footnotetext{
* Received by the editors 5 September 2007. Accepted for publication on 5 December 2007. Handling Editor: Harm Bart.

${ }^{\dagger}$ Department of Mathematics, Ben Gurion University of the Negev, P.0. Box 653, Beer-Sheva 84105, Israel (gilmi@cs.bgu.ac.il). Supported by the Kameah fund of the Israel.
} 
$|A|=\left(\left|a_{j k}\right|\right)_{j, l=1}^{n}$, i.e. $|A|$ is the matrix whose entries are absolute values of $A$ in $\left\{e_{k}\right\}_{k=1}^{n}$. The same sense has the symbol $|h|$ for a vector $h$. We write $C \geq 0$ if all the entries of a matrix $C$ in basis $\left\{e_{k}\right\}$ are nonnegative. If $C$ and $B$ are two real matrices, then we write $C \geq B$ if $C-B \geq 0$.

Clearly, $A=D+V$ where $D=\operatorname{diag}\left[a_{11}, \ldots, a_{n n}\right]$ is the diagonal and $V:=A-D$ is the off diagonal parts of $A$, respectively. That is, the entries $v_{j k}$ of $V$ are $v_{j k}=a_{j k}$ $(j \neq k)$ and $v_{j j}=0(j, k=1, \ldots, n)$. Denote by $r_{s}(C)$ the spectral radius of an operator $C$. Clearly,

$$
r_{s}(D)=\max _{j=1, \ldots, n}\left|a_{j j}\right| .
$$

Thanks to the well known inequality for the spectral radius [8, Section 16.5], [10], we have $r_{s}(A) \leq \tilde{r}_{A}$ where

$$
\tilde{r}_{A}:=\max _{j=1, \ldots, n} \sum_{k=1}^{n}\left|a_{j k}\right| .
$$

Denote by $\operatorname{co}(D)$ the closed convex hull of the diagonal entries $a_{11}, \ldots, a_{n n}$. Now we are in a position to formulate our main result.

THEOREM 1.1. Let $V$ be the off-diagonal part of an $n \times n$-matrix $A$. Let $f(\lambda)$ be holomorphic on a neighborhood of the circle $\Omega(A):=\left\{z \in \mathbb{C}:|z| \leq \tilde{r}_{A}\right\}$. Then with the notation

$$
\gamma_{k}(A):=\sup _{z \in \operatorname{co}(D)} \frac{\left|f^{(k)}(z)\right|}{k !}(k=0,1,2, \ldots)
$$

the inequality

$$
|f(A)| \leq \sum_{k=0}^{\infty} \gamma_{k}|V|^{k}
$$

holds, provided the series in (1.4) converges.

This theorem is proved in the next section.

Note that according to (1.2), we have the inequality

$$
r_{s}(|V|) \leq \max _{j=1, \ldots, n} \sum_{k=1, k \neq j}^{n}\left|a_{j k}\right| .
$$

A norm $\|\cdot\|$ in $\mathbb{C}^{n}$ is said to be ideal, if for all $h, g \in \mathbb{C}^{n}$ we have $\|h\| \leq\||h|\|$ and $\|h\| \leq\|g\|$, provided $|h| \leq|g|$. ¿From Theorem 1.1 it directly follows

Corollary 1.2. Let $f(\lambda)$ be holomorphic on a neighborhood of $\Omega(A)$. Let $\|$. be an arbitrary ideal norm in $\mathbb{C}^{n}$. Then

$$
\|f(A)\| \leq \sum_{k=0}^{\infty} \gamma_{k}\left\||V|^{k}\right\|
$$

provided the series in (1.5) converges. 
2. Proof of Theorem 1.1. By the equality $A=D+V$ we get

$$
\begin{gathered}
R_{\lambda}(A) \equiv(A-I \lambda)^{-1}=(D+V-\lambda I)^{-1}=\left(I+R_{\lambda}(D) V\right)^{-1} R_{\lambda}(D)= \\
R_{\lambda}(A)=\sum_{k=0}^{\infty}\left(R_{\lambda}(D) V\right)^{k}(-1)^{k} R_{\lambda}(D),
\end{gathered}
$$

provided the spectral radius $r_{0}(\lambda)$ of $R_{\lambda}(D) V$ is less than one. The entries of this matrix are

$$
\frac{a_{j k}}{\lambda_{j}-\lambda}\left(\lambda_{j}=a_{j j}, \lambda \neq a_{j j}, \quad j \neq k\right)
$$

and the diagonal entries are zero. Thanks to the above mentioned inequality for the spectral radius from [8], [10], we have

$$
r_{0}(\lambda) \leq \max _{j} \sum_{k=1, k \neq j}^{n} \frac{\left|a_{j k}\right|}{\left|a_{j j}-\lambda\right|} .
$$

So the inequality

$$
|\lambda|>\tilde{r}_{A}=\max _{j} \sum_{k=1}^{n}\left|a_{j k}\right|,
$$

implies that $r_{0}(\lambda)<1$ and the series

$$
\sum_{k=0}^{\infty}\left(R_{\lambda}(D) V\right)^{k}(-1)^{k}
$$

converges. Thus

$$
f(A)=-\frac{1}{2 \pi i} \int_{|\lambda|=r} f(\lambda) R_{\lambda}(A) d \lambda=\sum_{k=0}^{\infty} C_{k}\left(r=\tilde{r}_{A}+\epsilon, \epsilon>0\right),
$$

where

$$
C_{k}=(-1)^{k+1} \frac{1}{2 \pi i} \int_{|\lambda|=r} f(\lambda)\left(R_{\lambda}(D) V\right)^{k} R_{\lambda}(D) d \lambda .
$$

Since $D$ is a diagonal matrix with respect to basis $\left\{e_{k}\right\}$, we can write out

$$
R_{\lambda}(D)=\sum_{j=1}^{n} \frac{Q_{j}}{\lambda_{j}-\lambda}
$$

where $Q_{k}=\left(., e_{k}\right) e_{k}$. We thus have

$$
C_{k}=\sum_{j_{1}=1}^{n} Q_{j_{1}} V \sum_{j_{2}=1}^{n} Q_{j_{2}} V \ldots V \sum_{j_{k}=1}^{n} Q_{j_{k}} I_{j_{1} j_{2} \ldots j_{k+1}} .
$$


Here

$$
I_{j_{1} \ldots j_{k+1}}=\frac{(-1)^{k+1}}{2 \pi i} \int_{|\lambda|=r} \frac{f(\lambda) d \lambda}{\left(\lambda_{j_{1}}-\lambda\right) \ldots\left(\lambda_{j_{k+1}}-\lambda\right)} .
$$

Lemma 1.5.1 from [7] gives us the inequalities

$$
\left|I_{j_{1} \ldots j_{k+1}}\right| \leq \gamma_{k}\left(j_{1}, j_{2}, \ldots, j_{k+1}=1, \ldots, n\right) .
$$

Hence, by (2.2)

$$
\left|C_{k}\right| \leq \gamma_{k} \sum_{j_{1}=1}^{n} Q_{j_{1}}|V| \sum_{j_{2}=1}^{n} Q_{j_{2}}|V| \ldots|V| \sum_{j_{k}=1}^{n} Q_{j_{k}} .
$$

But

$$
\sum_{j_{1}=1}^{n} Q_{j_{1}}|V| \sum_{j_{2}=1}^{n} Q_{j_{2}}|V| \ldots|V| \sum_{j_{k}=1}^{n} Q_{j_{k}}=|V|^{k} .
$$

Thus

$$
C_{k} \leq \gamma_{k}|V|^{k}
$$

Now (2.1) implies

$$
|f(A)| \leq \sum_{k=0}^{\infty}\left|C_{k}\right| \leq \sum_{k=0}^{\infty} \gamma_{k}|V|^{k}
$$

As claimed.

\section{Examples and applications of Theorem 1.1.}

EXAMPle 3.1. Let $f(A)=A^{m} \quad(m=1,2, \ldots)$. Then

$$
f^{(k)}(\lambda)=\frac{m !}{(m-k) !} \lambda^{m-k}, \gamma_{k}=\frac{m !}{(m-k) ! k !} r_{s}^{m-k}(D) .
$$

So

$$
\left|A^{m}\right| \leq \sum_{k=0}^{m} \frac{m !}{(m-k) ! k !} r_{s}^{m-k}(D)|V|^{k}=\left(r_{s}(D) I+|V|\right)^{m} .
$$

This inequality can be directly obtained from the equality $A=D+V$. It only illustrates Theorem 1.1.

EXAMPle 3.2. Let $f(A)=e^{A t}(t \geq 0)$. Then

$$
f^{(k)}(\lambda)=t^{k} e^{\lambda t} ; \gamma_{k}=\frac{t^{k}}{k !} e^{\alpha(D) t}
$$


where $\alpha(D)=\max _{k} R e a_{k k}$. So

$$
e^{A t} \leq e^{\alpha(D) t} \sum_{k=0}^{\infty} \frac{t^{k}}{k !}|V|^{k}=e^{(\alpha(D) I+|V|) t}(t \geq 0) .
$$

Example 3.3. Let $f(A)=\sin (A t)(t \geq 0)$. Then

$$
f^{(2 k)}(\lambda)=t^{2 k}(-1)^{k} \sin (\lambda t) ; f^{(2 k+1)}(\lambda)=t^{2 k}(-1)^{k} \cos (\lambda t) .
$$

Let the diagonal $D$ be real, then $\gamma_{k} \leq \frac{1}{k !} t^{k}$. So

$$
|\sin (A t)| \leq \sum_{k=0}^{\infty} \frac{1}{k !} t^{k}|V|^{k}=e^{t|V|} .
$$

Consider the second order nonlinear differential equation

$$
\begin{aligned}
\frac{d^{2} x}{d t^{2}}+A^{2} x(t) & =F(x(t)) \quad(t>0) \\
\frac{d x(0)}{d t} & =x(0)=0
\end{aligned}
$$

where $A$ is a real matrix, $F: \mathbb{R}^{n} \rightarrow \mathbb{R}^{n}$ is a continuous function, satisfying

$$
\|F(h)\| \leq v+q\|h\| \quad\left(v, q=\text { cons } ; h \in \mathbb{C}^{n}\right)
$$

with some ideal norm. Problem (3.1), (3.2) is equivalent to the following equation:

$$
x(t)=\int_{0}^{t} \sin A(t-s) F(x(s)) d s .
$$

So

$$
\|x(t)\| \leq \int_{0}^{t}\|\sin A(t-s)\|(q\|x(s)\|+v) d s
$$

with an arbitrary ideal norm. Put $y(t)=\|x(t)\|$. Then by Example 3.3,

$$
y(t) \leq v f(t)+q \int_{0}^{t} e^{\||V|\|(t-s)} y(s) d s
$$

where

$$
f(t)=\int_{0}^{t} e^{\|\| V \mid \| s} d s
$$

Hence taking into account that $f$ monotonically increases, we get by the Gronwall inequality,

$$
y(t) \leq v f(t) \exp [q f(t)] .
$$

Such estimates are important, in particular, in the theory of oscillations, cf. [1]. 
4. Infinite matrices. Let $l^{p}(p \geq 1)$ be a complex Banach space of sequences $x=\left\{x_{k} \in \mathbb{C}\right\}_{k=1}^{\infty}$ with the norm

$$
\begin{gathered}
\|x\|_{p}=\left(\sum_{k=1}^{\infty}\left|x_{k}\right|^{p}\right)^{1 / p}(1 \leq p<\infty), \\
\|x\|_{\infty}=\sup _{k}\left|x_{k}\right| .
\end{gathered}
$$

Let $A=\left(a_{j k}\right)_{k=1}^{\infty}$ be an infinite matrix, $|A|=\left(\left|a_{j k}\right|\right)_{j, k=1}^{\infty}$ and $|x|=\left\{\left|x_{k}\right|\right\}$ for an $x=\left\{x_{k}\right\} \in l^{p}$. The sense of the symbols $\geq, \leq$ for vectors and matrices is the same as in the finite dimensional case. Again $D=\operatorname{diag}\left[a_{11}, a_{22}, \ldots\right]$ and $V:=A-D$ are the diagonal and off diagonal parts of $A$, respectively. Matrix valued function $f(A)$ is defined as in (1.1) and $c o(D)$ is the closed convex hull of the infinite set of the diagonal entries $\left\{a_{j j}\right\}$.

Assume that

$$
\tilde{r}_{A}=\sup _{j=1,2, \ldots} \sum_{k=1}^{\infty}\left|a_{j k}\right|<\infty,
$$

then as in the finite dimensional case, $r_{s}(A) \leq \tilde{r}_{A}$, cf. [8]. Hence it follows that

$$
r_{s}(|V|) \leq \sup _{j=1,2, \ldots} \sum_{k=1, k \neq j}^{\infty}\left|a_{j k}\right| .
$$

Moreover, it is not hard to check that under condition (4.1), matrix $A=\left(a_{j k}\right)$ generates a bounded linear operator in $l^{p}$ for any $p \geq 1$.

THEOREM 4.1. Let an infinite matrix $A=\left(a_{j k}\right)$ satisfy condition (4.1) and $f(\lambda)$ be holomorphic on a neighborhood of the circle $\Omega(A):=\left\{z \in \mathbb{C}:|z| \leq \tilde{r}_{A}\right\}$. Let $\gamma_{k}$ be defined as in (1.3). Then inequality (1.4) is valid, provided the series in (1.4) converges in the norm of $l^{\infty}$.

Proof. Let $P_{n}$ be the projection onto subspace generated by the first $n$ elements of the standard basis. Then the finite dimensional matrices $A_{n}=P_{n} A P_{n}$ strongly converge to $A$. Let $D_{n}=P_{n} D$ and $V_{n}=P_{n} V P_{n}$ be the diagonal and off-diagonal parts of $A_{n}$, respectively. Then by Theorem 1.1,

$$
\left|f\left(A_{n}\right)\right| \leq \sum_{k=0}^{\infty} \gamma_{k}\left|V_{n}\right|^{k} \leq \sum_{k=0}^{\infty} \gamma_{k}|V|^{k} .
$$

But $f\left(A_{n}\right) \rightarrow f(A)$ in the strong topology of $l^{\infty}[2]$. So each entry of $f\left(A_{n}\right)$ converges to the corresponding entry of $f(A)$. This proves the result.

Corollary 4.2. Let condition (4.1) hold and $f(\lambda)$ be holomorphic on a neighborhood of $\Omega(A)$. Then for any $p \geq 1$ we have

$$
\|f(A)\|_{p} \leq \sum_{k=0}^{\infty} \gamma_{k}\left\||V|^{k}\right\|_{p}
$$


provided the series in (4.2) converges.

\section{REFERENCES}

[1] Yu L. Daleckii and M.G. Krein. Stability of Solutions of Differential Equations in Banach Space. American Mathematical Society, Providence, R.I., 1974.

[2] N. Dunford and J.T. Schwartz. Linear Operators, part I, Wiley-Interscience, New York, 1966.

[3] B. Fritzsche, B. Kirstein, and A. Lasarow. On Schwarz-Pick-Potapov block matrices of matrix-valued functions which belong to the extended Potapov class. Analysis (Munich), 22(3):243-263, 2002.

[4] B. Fritzsche, B. Kirstein, and A. Lasarow. Orthogonal rational matrix-valued functions on the unit circle: Recurrence relations and a Favard-type theorem. Math. Nachr., 279:513-542, 2006.

[5] I.M. Gel'fand and G.E. Shilov. Some Questions of Theory of Differential Equations. Nauka, Moscow, 1958 (in Russian).

[6] M.I. Gil'. Estimates for norm of matrix-valued functions. Linear Multilinear Algebra, 35:65-73, 1993.

[7] I.M. Gil'. Operator Functions and Localization of Spectra. Lectures Notes In Mathematics vol. 1830, Springer-Verlag, Berlin, 2003.

[8] M.A. Krasnosel'skii, J. Lifshits, and A. Sobolev. Positive Linear Systems. The Method of Positive Operators. Heldermann Verlag, 1983.

[9] A. Lasarow. Dual Szegö pairs of sequences of rational matrix-valued functions. Int. J. Math. Math. Sci. 2006, No. 5, 23723, 37 p., 2006.

[10] M. Marcus and H. Minc. A Survey of Matrix Theory and Matrix Inequalities. Allyn and Bacon, Boston, 1964.

[11] L. Verde-Star. Functions of matrices. Linear Algebra Appl., 406:285-300, 2005. 